\title{
Effects of Solvation of 2-Methylpyridine and 2,6-Dimethylpyridine in Dilute Solutions in Water and Methanol on the Limiting Partial Molar Compressibility and Volume
}

\begin{abstract}
B. Czech and W. MarczaK
Institute of Chemistry, University of Silesia, Szkolna 9, 40-006 Katowice, Poland

The limiting partial molar volumes and isentropic compressibilities of 2-methylpyridine and 2,6-dimethylpyridine in aqueous and methanolic solutions were calculated from the densities and speeds of sound at 293.15 and $298.15 \mathrm{~K}$. All the limiting functions are smaller than the respective functions for the pure amines, due to the hydrogen bonds $\mathrm{O}-\mathrm{H}$. . . N between the amine molecules and those of water or methanol. The standard functions of transfer of the amines from methanol to water are negative which results from different nature of interactions in the aqueous and methanolic solutions. Most probably, the hydrophobic effect contributes to the limiting partial volumes and compressibilities of the amines diluted in water. That makes them smaller than the respective functions for the amines in methanol.
\end{abstract}

PACS numbers: 82.60.Lf

\section{Introduction}

The properties of water and aqueous solutions have been extensively studied due to the unique role of water in biological systems. There are cogent empirical evidences for the association of water molecules into dynamic, three-dimensional structures in the liquid phase. Solutes may reinforce or weaken these quasi-crystalline lattices. The solute-water interactions are often discussed in terms of the hydrophilic and hydrophobic hydration. These phenomena influence the properties of aqueous solutions. Methanol, similarly to water, is an associated liquid. However, its molecules do not form three-dimensional lattices but linear or cyclic associates in the pure liquid phase and in the mixtures. This difference is reflected in the thermodynamic properties of the two systems. 
In the present study, we compared dilute binary solutions of 2-methylpyridine and 2,6-dimethylpyridine in water and methanol. The methylpyridines are cyclic compounds with molecules having nitrogen atom in the aromatic ring (Fig. 1). The nitrogen atom has one lone pair of electrons and it is capable of forming the hydrogen bond with a proton-donating molecule. Thus, a molecule of water or methanol can be attached to the ring through the $\mathrm{O}-\mathrm{H} . . \mathrm{N}$ bonds. The cross-associates formed in this way can act as a proton-acceptor in further hydrogen bonding due to the two lone pairs of electrons located on the oxygen atom of the water or methanol molecule. Furthermore, the associates containing water may participate in the three-dimensional aqueous lattice as donors of proton. The formation of a cage-like structure around the molecules of 2-methylpyridine in water has been suggested earlier [1]. Similar arrangement of molecules is obviously impossible in the methanolic solutions. Different solvation shells in the aqueous and methanolic systems should lead to non-zero values of the functions of transfer.
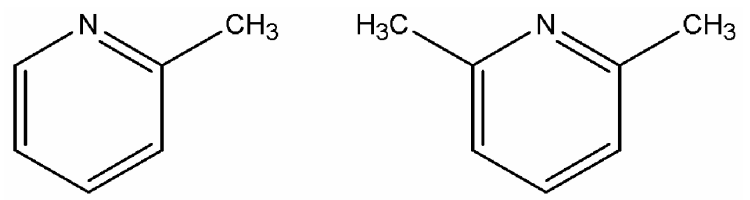

Fig. 1. The molecules of 2-methylpyridine (left) and 2,6-dimethylpyridine (right).

The standard functions of transfer represent a measure of the difference in the coupling work of the solute with the considered solvents, and are defined in the following way:

$$
E^{i \rightarrow j}=-E^{j \rightarrow i}=E^{\infty}(j)-E^{\infty}(i)
$$

where $E^{\infty}(i)$ and $E^{\infty}(j)$ are the partial molar quantities of the solute infinitely dilute in the solvents $i$ and $j$, and $E$ is an extensive thermodynamic function, e.g. volume, enthalpy, entropy, etc. In the studies of aqueous systems, commonly discussed are the standard functions of transfer of a solute molecule from a nonelectrolyte solvent to water [2].

\section{Results}

Speeds of ultrasound, $u$, and densities, $\rho$, of the binary systems 2-methylpyridine + methanol and 2,6-dimethylpyridine + methanol have been measured using a sing-around meter designed and constructed in our laboratory [3], and a vibrating-tube densimeter Anton Paar DMA 5000. The experimental procedure has been described in detail previously $[4,5]$. As the raw data are extensive, since they cover the whole concentration range rather than infinitely dilute solutions discussed here, they will be published elsewhere. The speeds and densities for the aqueous systems have been taken from our earlier papers $[1,4,6]$. 
All the calculations were made for the temperatures 293.15 and 298.15 K. For those temperatures, the necessary experimental data are available. The temperature interval of $5 \mathrm{~K}$, although rather narrow, proved to be wide enough for tracing the changes in the partial functions.

TABLE

The partial molar volumes and isentropic compressibilities of 2-methylpyridine and 2,6-dimethylpyridine as pure liquids and in the infinitely dilute solutions in methanol and water.

\begin{tabular}{l|c|c|c|c}
\hline \hline for $V^{\prime} \mathrm{s}\left[\mathrm{m}^{3} \mathrm{~mol}^{-1}\right]$ & \multicolumn{2}{|c|}{ 2-Methylpyridine } & \multicolumn{2}{|c}{ 2,6-Dimethylpyridine } \\
\cline { 2 - 5 } for $K$ 's $\left[\mathrm{m}^{5} \mathrm{~N}^{-1} \mathrm{~mol}^{-1}\right]$ & $T=293.15 \mathrm{~K}$ & $T=298.15 \mathrm{~K}$ & $T=293.15 \mathrm{~K}$ & $T=298.15 \mathrm{~K}$ \\
\hline$V_{\mathrm{a}}^{\infty} \times 10^{6}$ & 98.645 & 99.136 & 116.151 & 116.719 \\
$V_{\mathrm{a}}^{\infty}\left(\mathrm{CH}_{3} \mathrm{OH}\right) \times 10^{6}$ & 94.428 & 94.889 & 110.284 & 110.807 \\
$V_{\mathrm{a}}^{\infty}\left(\mathrm{H}_{2} \mathrm{O}\right) \times 10^{6}$ & 93.092 & 93.668 & 107.641 & 108.431 \\
$K_{\mathrm{a}}^{0} \times 10^{14}$ & 5.326 & 5.541 & 6.591 & 6.863 \\
$K_{\mathrm{a}}^{\infty}\left(\mathrm{CH}_{3} \mathrm{OH}\right) \times 10^{14}$ & 1.270 & 1.304 & 1.905 & 1.971 \\
$K_{\mathrm{a}}^{\infty}\left(\mathrm{H}_{2} \mathrm{O}\right) \times 10^{14}$ & 0.622 & 1.099 & 0.647 & 1.225
\end{tabular}
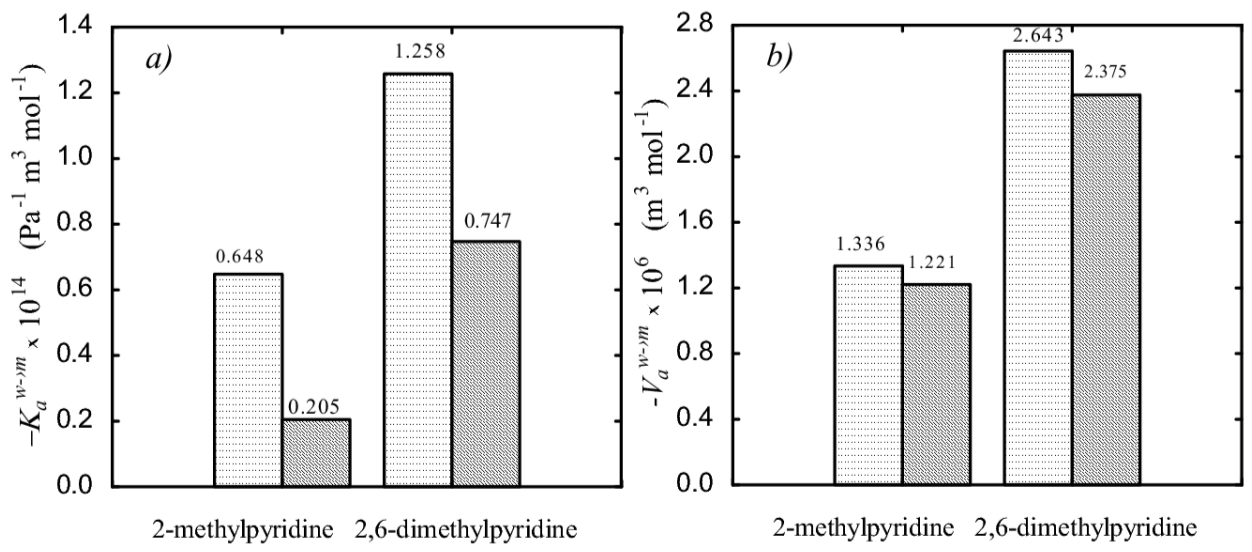

Fig. 2. The standard molar functions of transfer of the methylpyridines from methanol to water: (a) isentropic compressibility, (b) volume; light bars - $293.15 \mathrm{~K}$, dark bars $-298.15 \mathrm{~K}$.

The molar volumes, $V$, and the molar isentropic compressibilities, $K \equiv$ $-(\delta V / \delta p)_{S}$, were calculated according to the well-known relationships

$$
\begin{aligned}
& V=\left(M_{\mathrm{a}} x_{\mathrm{a}}+M_{\mathrm{s}} x_{\mathrm{s}}\right) / \rho, \\
& K=V \kappa,
\end{aligned}
$$

where $M$ is the molecular mass, $x$ is the mole fraction, and $\kappa \equiv-V^{-1}(\delta V / \delta p)_{S}$ 
is the isentropic compressibility. The subscripts "a" and "s" stand for the amine (2-methylpyridine or 2,6-dimethylpyridine) and the solute (water or methanol), respectively. The isentropic compressibility was calculated from the Laplace formula

$$
\kappa=\left(\rho u^{2}\right)^{-1} .
$$

The limiting partial molar functions of the amines in binary solutions were calculated using the following equation:

$$
E_{\mathrm{a}}^{\infty}=\lim _{x_{\mathrm{a}} \rightarrow 0} E_{\mathrm{a}},
$$

where

$$
\begin{aligned}
& E_{\mathrm{a}}=E+\left(1-x_{\mathrm{a}}\right)\left(\delta E / \delta x_{\mathrm{a}}\right), \\
& E=E_{\mathrm{a}}^{0} x_{\mathrm{a}}+E_{\mathrm{s}}^{0}\left(1-x_{\mathrm{a}}\right)+x_{\mathrm{a}}\left(1-x_{\mathrm{a}}\right) \sum_{i=0}^{n} a_{i}\left(1-2 x_{\mathrm{a}}\right)^{i} .
\end{aligned}
$$

In the latter formulae, $E$ is the molar volume $(V)$ or compressibility $(K), a_{i}$ are empirical coefficients obtained by the least squares method, and the superscript " 0 " denotes the pure substance. Finally, the standard volumes and isentropic compressibilities of transfer of the amines from methanol to water were calculated according to Eq. (1). The results are reported in Table and plotted in Fig. 2.

\section{Discussion and conclusions}

Binary mixtures of the methylpyridines with water and methanol are thermodynamically non-ideal systems. This non-ideality results from different sizes and shapes of the molecules as well as from the formation of the mixed associates due to $\mathrm{O}-\mathrm{H} \ldots \mathrm{N}$ bonds. In consequence, the mean volume per one molecule of the amine decreases when it is diluted in methanol: the limiting partial molar volume of 2-methylpyridine in methanol is by $c a .4 \mathrm{~cm}^{3} \mathrm{~mol}^{-1}$ smaller than the molar volume of pure 2-methylpyridine. The difference for 2,6-dimethylpyridine reaches $6 \mathrm{~cm}^{3} \mathrm{~mol}^{-1}$ (Table). Larger effects accompany dilution in water. They are close to 5.5 and $8.5 \mathrm{~cm}^{3} \mathrm{~mol}^{-1}$ for 2-methylpyridine and 2,6-dimethylpyridine, respectively. Consequently, the transfer of the methylpyridines from methanol to water leads to the decrease in the partial volumes, i.e. the volumes of transfer are negative (Fig. 2).

The decrease in partial isentropic compressibility of the amines that accompanies the dissolving is much more spectacular. In the methanolic solutions at $293.15 \mathrm{~K}$, the limiting partial compressibilities amount $24 \%$ and $28 \%$ of the values for the pure 2-methylpyridine and 2,6-dimethylpyridine. The respective values for the aqueous solutions are just $12 \%$ and $10 \%$. Similar change is smaller at $298.15 \mathrm{~K}$, but still profound. Thus, the substitution of the methanolic solvation shell by the aqueous one causes that both the partial volume and compressibility of the methylpyridines decrease. The effects are bigger for 2,6-dimethylpyridine than 2-methylpyridine most probably because of stronger bonding of the former 
to the proton-donating molecules. That supposition is supported by the convincing results of theoretical and empirical studies. The association energies in the isolated 1:1 complexes of pyridine and its methyl derivatives with water have been calculated theoretically by Pápai and Jancsó using the Møller-Plesset perturbation theory [7]. For 2,6-dimethylpyridine and 2-methylpyridine, these energies are $21.2 \mathrm{~kJ} \mathrm{~mol}^{-1}$ and $20.0 \mathrm{~kJ} \mathrm{~mol}^{-1}$, respectively. The negative enthalpy and entropy of solution of 2,6-dimethylpyridine in water are also higher than those of 2-methylpyridine. The enthalpies are $-15.90 \mathrm{~kJ} \mathrm{~mol}^{-1}$ and $-12.64 \mathrm{~kJ} \mathrm{~mol}^{-1}$, and entropies $-89.5 \mathrm{~J} \mathrm{~K}^{-1} \mathrm{~mol}^{-1}$ and $-72.4 \mathrm{~J} \mathrm{~K}^{-1} \mathrm{~mol}^{-1}[8]$.

Although the molecule of 2,6-dimethylpyridine is bigger than that of 2-methylpyridine, the hydrophobic hydration shells of the two solutes are probably similar one to another. That is evidenced by very close dielectric relaxation times in the two binary solutions, approximately equal to $12 \mathrm{ps}$ [9]. With increasing temperature, the three-dimensional structure of water decays and the two solvents, water and methanol, become more similar. Changes in the partial compressibility and volume, induced by temperature, are larger for the amines in aqueous solutions than for those in the methanolic ones. As a result, the negative functions of transfer decrease (Fig. 2).

The results reported have shown that although the $\mathrm{O}-\mathrm{H} . . . \mathrm{N}$ hydrogen bonds determine the properties of the methylpyridine solutions in water and methanol, their energy is by no means the only factor that has to be considered. The hydrophobic effect cannot be neglected, especially at lower temperatures. Although the role of the interactions between the apolar regions and the hydration shell is important [7], there are still no reliable results of theoretical calculations that could precisely elucidate the behavior of a few dozen of molecules that form the shell. Another question of great importance is the propensity of pyridine and its methyl-derivatives to self-association in the pure state and in solutions. The UV spectroscopy studies of aqueous solutions pointed to the association-favoring influence of the methyl groups substituted in the ortho position in the ring [10]. Contrary to that, the results of thermodynamic experiments were interpreted assuming the degree of self-association decreasing in the order: pyridine - 2-methylpyridine 2,6-dimethylpyridine $[8,11]$.

\section{References}

[1] S. Ernst, W. Marczak, Bull. Acad. Pol. Sci. Chem. 43, 259 (1995).

[2] F. Franks, Water, The Royal Society of Chemistry, Cambridge 1983.

[3] S. Ernst, W. Marczak, R. Manikowski, E. Zorȩbski, M. Zorębski, Acoust. Lett. 15, $123(1992)$.

[4] S. Ernst, W. Marczak, D. Kmiotek, J. Chem. Eng. Data 41, 128 (1996).

[5] W. Marczak, J. Chem. Eng. Data 44, 621 (1999).

[6] S. Ernst, W. Marczak, A. Kądziołka, Bull. Acad. Pol. Sci. Chem. 42, 83 (1994).

[7] I. Pápai, G. Jancsó, J. Phys. Chem. A 104, 2132 (2000). 
[8] L. Sacconi, P. Paoletti, M. Ciampolini, J. Am. Chem. Soc. 82, 3828 (1960).

[9] U. Kaatze, Chr. Neumann, R. Pottel, J. Solution Chem. 16, 191 (1987).

[10] F. Peral, E. Gallego, J. Mol. Struct. 326, 59 (1994).

[11] J. Abe, K. Nakanishi, H. Touhara, J. Chem. Thermodyn. 10, 483 (1978). 